\title{
Del pasado al futuro: anotaciones feministas para una ciencia democrática'
}

\section{RESUMEN}

Carmen Magallón realiza en este importante artículo un breve análisis sobre el aporte que las mujeres han brindado en la historia de la ciencia. Señala que en la educación y en la práctica, conocer al pasado y transmitirlo es una actividad poco valorada.

Es a través de la historia donde se puede aprender a valorar la influencia de los factores sociales en el conocimiento, a conocer las circunstancias que favorecieron la participación o la exclusión de los distintos grupos humanos en el desarrollo científico. Las mujeres científicas han sido mucho más de lo que imaginamos, a pesar de los obstáculos que prohibían su participación en las instituciones de la ciencia.

Palabras clave: Pasado, ciencia, teorías, tecnociencia, feministas.

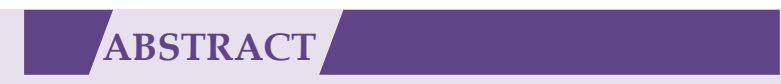

Carmen Magallón carries out in this important paper a brief analysis on the contribution that women have given in the history of science. She points out that in education and practice, to know the past and transfer it is an unappreciated activity.

It is through history where can learn to appreciate the influence of social factors on knowledge, to know the circumstances that favored the participation or exclusion of different human groups in scientific development. The women scientists have been much more than one imagines, despite the obstacles that prohibited their participation in institutions of science.

Keywords: Past, science, theories, technoscience, feminist.
En la educación y en la práctica científica, evocar el pasado, conocerlo y transmitirlo es una actividad poco valorada. La enseñanza académica de la ciencia se limita a potenciar las destrezas que permiten entender y manejar modelos matemáticos sobre el mundo, modelos que fueron construidos por grandes personajes que quedaron esclerotizados en los libros de texto, reducidos a sus propias elaboraciones. ${ }^{3}$ Se pone el acento en la transmisión-asimilación de un inmenso bagaje de conocimientos, así como en la familiarización y puesta en práctica de los métodos que permiten que este bagaje siga creciendo. Mientras, reflexionar sobre quiénes fueron los protagonistas de esta empresa, cómo y en qué circunstancias se produjo este conocimiento, asî como el estatus del mismo y las repercusiones sociales de que la investigación se oriente en un sentido o en otro, adquiere, en esta práctica, un carácter secundario. Nada que se parezca a una mínima reflexión epistemológica.
Ninguna mención a las polémicas habidas a lo largo de la historia de la ciencia. Desconocimiento total acerca de la significación y los debates que, por ejemplo, interesaron a los integrantes del Círculo de Viena -muchos de ellos de formación inicial en ciencia-; acerca de qué sea la Concepción Heredada o el falsacionismo de Popper, las revoluciones científicas de Thomas Kuhn, los programas de investigación de Lakatos, las críticas a los dogmas del empirismo de Quine o la disertación contra el método de Feyerabend.

Este desdén por la autorreflexión sobre el propio proceder y sobre los productos que se derivan, por la interrogación acerca de los aspectos contextuales que influyen y son influidos por la empresa científica, en suma por la filosofía y la historia de la ciencia, entra en contradicción, contrasta, con la actitud crítica que se exige para el propio quehacer científico. Además, dado

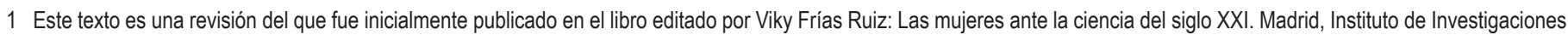
Feministas, Universidad Complutense, 213-225, 2001.

2 Seminario Interdisciplinar de Estudios de la Mujer (SIEM), Universidad de Zaragoza.

3 Sin biografía, sin familia, despojados de su contexto histórico-social. 


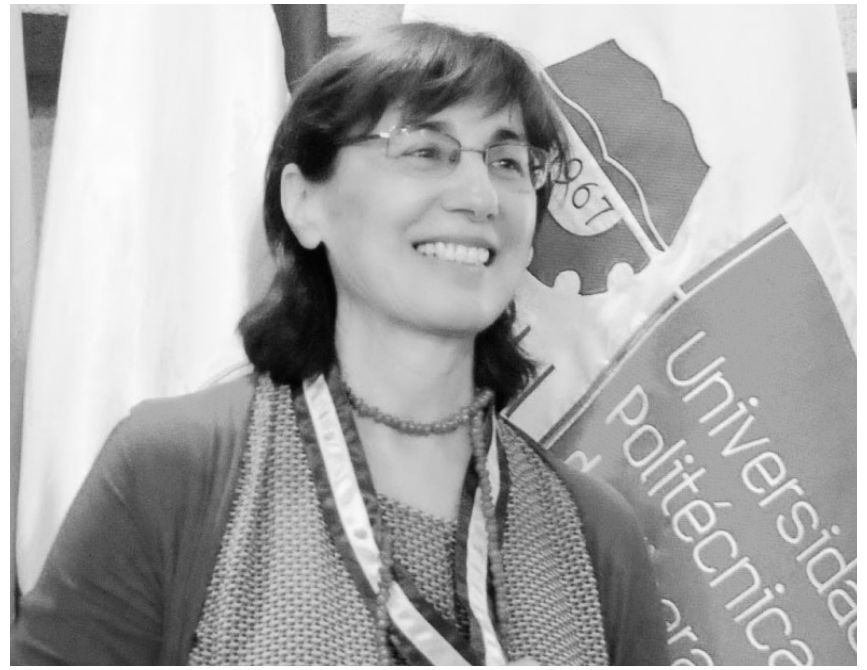

Dra. Carmen Magallón Portolés/Foto: Relaciones Públicas UPOLI.

el poder de conformar nuestras vidas que tiene la ciencia, sobre todo en su fase actual de tecnociencia, desconocer el estatus, alcance, límites y sesgos de ese conocimiento, pasa a ser una laguna grave.

En el pasado residen restos de libertad que son necesarios y significativos para la existencia de un presente y un futuro más libres. En el devenir de la ciencia, mirar hacia el pasado nos permite conocer las raíces, saber de dónde venimos, airear los lastres que arrastramos, todo lo cual permite explicar mejor el presente. Es en la historia donde podemos aprender a valorar la influencia de los factores sociales en el conocimiento, a conocer las circunstancias que favorecieron la participación o la exclusión de los distintos grupos humanos en el desarrollo científico.

En el caso de las mujeres, si se mira el pasado desde un sistema de referencia que toma en consideración su plural experiencia, nos damos cuenta que en la historia de la ciencia ha habido más científicas de las que en general son conocidas. No sólo existió Madame Curie. Y aunque hubo obstáculos y barreras que se levantaron para prohibir su participación en las instituciones de la ciencia, lo cierto es que siempre hubo mujeres que poseyeron autoridad científica en su época, científicas sobresalientes como Hipatia de Alejandría, Hildegarda de Bingen o Madame de Chatelet, por ejemplo; y también hubo otras que elaboraron conocimiento e hicieron ciencia en grupos y redes de intercambio y solidaridad.
Durante un tiempo empeñé mis esfuerzos en la identificación y visibilización de las españolas que se dedicaron a la ciencia a lo largo del primer tercio del siglo $X X$, recalando en particular en aquellas que trabajaron en las ciencias físico-químicas, dos campos por entonces estrechamente entrelazados. En esa época, en España, eran pocas las mujeres que se adentraban por los caminos de los estudios universitarios. Su número fue creciendo hasta el apogeo que se dio en los años 30, durante el periodo republicano anterior a la Guerra Civil. Pese a lo que pueda parecer, desde 1910 hasta 1936, el ritmo con el que crecían las que se encaminaban a carreras de ciencias fue mayor que el de aquellas que se dirigían al resto de las carreras. Es ésta una de las conclusiones del estudio, que ha cuajado en el libro Pioneras españolas en las ciencias. ${ }^{4}$

Esta conclusión, que no significa un mayor número, en valores absolutos, de estudiantes universitarias españolas de principios de siglo $X X$ en las facultades de ciencias, sino una tendencia marcada por un porcentaje de mujeres en estas facultades que creció de manera más rápida que en el conjunto de la universidad y que contrasta con una idea preconcebida diferente bastante arraigada.

Es importante asumir que la experiencia de estas mujeres tiene un sentido para nuestra propia experiencia como profesionales de la ciencia. Ellas son las pioneras más cercanas, geográfica y culturalmente. Debido a los avatares de la Guerra Civil española, su práctica científica se vio rápidamente truncada en el tiempo, pero esto no resta un ápice al significado simbólico que posee este grupo para las generaciones de mujeres posteriores. Por una parte, su presencia y actividad en espacios antes concebidos como sólo de varones supuso un hito en el camino de las mujeres hacia la condición de iguales; por otra, su experiencia fragmentada, es decir esa existencia que dio cabida a tramos de vida innovadores seguidos o solapados con la asunción de roles tradicionales, es todavía un espejo en el que podemos observar las inadecuaciones históricas y sociales que impiden transformar las diferencias humanas en multiplicidad y riqueza, y obligan a optar de modo reduccionista entre homogeneización o desigualdad.

4 MAGALLÓN PORTOLÉS, C. (1998 y 2004) Pioneras españolas en las ciencias. Las mujeres del Instituto Nacional de Físíca y Química, Madrid, CSIC. Puede consultarse 0 descargarse totalmente en el siguiente enlace: https:/www.academia.edu/1464693/_2004_Pioneras_espa\%C3\%B1olas_en_las_ciencias_1a_edici\%C3\%B3n_de_1998. 


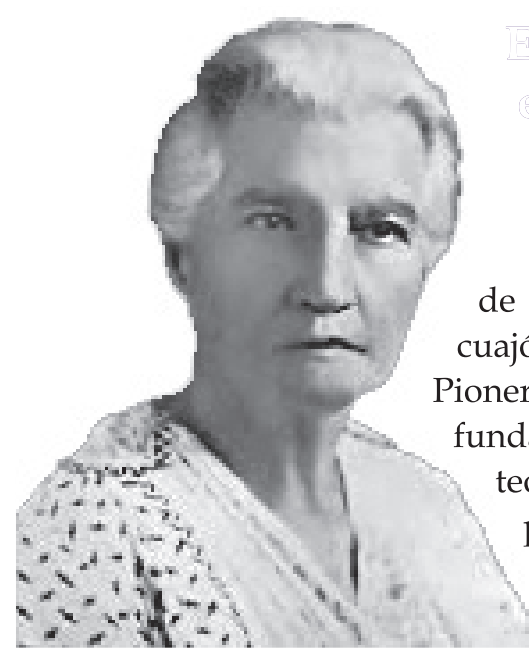

Josefa Toledo de Aguerri (1866-1962). Primera educadora en Nicaragua, feminista pionera nicaragüense, ganadora del reconocimiento continental de "Mujer de las Américas", antes se le había otorgado a la Premio Nobel de la Paz Gabriela Mistral, Eleanor Roosevelt, Minerva Bernardino y Carrie Capman.

recuperar el protagonismo de las mujeres en la ciencia, pasado y presente, tanto el protagonismo individual -las excepcionales- como el protagonismo de grupo - las que formaron o forman parte de las comunidades científicas, codo a codo con los varones de su tiempo-.

Con las herramientas críticas que nos brindaron pudimos 'verlas', ver a estas mujeres de ciencias que antes no había 'visto' nadie. Aprendimos también que hay que ir más allá. Que además de rescatar la parte de protagonismo que corresponde a las mujeres, en la construcción de la ciencia, hay que revisar lo que se entiende por ciencia, ver si en la transmisión científica se han hurtado actividades y conocimientos que no encajaban en el esquema de la corriente principal, y si la pretendida objetividad que proclama es tal o si, como empresa humana, lleva en sí misma huellas/sesgos de género (y otros) que ponen en cuestión el carácter universal que se autoatribuye.

Las reflexiones epistemológicas nutren la orientación de los estudios de género y ciencia. En una constante profundización permiten conectar las vías de exclusión social con el contenido del conocimiento y pasar del problema de las mujeres en la ciencia al problema de la ciencia en el feminismo (HARDING, 1986).

Para Elena Grau, ${ }^{5}$ "la osadía de las mujeres que han dudado de la ciencia masculina y se han interrogado sobre la misma ha sido no sólo la de decir y cuestionar el hecho de que la científica es una institución sociológicamente masculina, sino que el conocimiento que en ella se produce responde a intereses y a interrogantes planteados por sólo uno de los dos sujetos que componen la especie humana, el hombre, y que la metodología que emplea para validar el conocimiento obtenido a partir de estos interrogantes tiene que ver con la forma que el sexo masculino tiene de relacionarse en el mundo y con la naturaleza. Es decir estas mujeres han hecho parcial, al atribuirlo al hacer de uno de los dos sexos, lo que parecía ser el conocimiento más objetivo y universal posible y, por tanto, el más verdadero" ${ }^{\prime \prime}$.

Las epistemologías feministas han dado diferentes respuestas a la pregunta de por qué el desarrollo de los estudios de las mujeres ha conducido a la obtención de un saber que es significativamente mejor que el saber previo. Las ciencias sociales, la biología, la psicología, la historia de la ciencia y la medicina, entre otras, se han beneficiado de los resultados obtenidos por investigadoras que criticaron el conocimiento asentado en sus disciplinas. ${ }^{7}$ Puesto que los trabajos a que hago referencia nacen al calor del movimiento feminista y las reivindicaciones políticas que lo acompañan, la pregunta que surge es por qué, o a qué es debido que un conocimiento enraizado en un movimiento político sea capaz de proporcionar resultados menos distorsionados que el obtenido a partir de presupuestos pretendidamente neutros. Una pregunta que las filósofas e historiadoras feministas de la ciencia han explicado cabalmente.

Es muy difícil que alguien formado en una tradición positivista, como la que todavía predomina en nuestras facultades de ciencias, pueda adoptar enfoques científicos -sociales o naturales- no positivistas, si no

5 Miembro del grupo de la revista En pie de Paz, forma parte del Institut Català Internacional per la Pau (ICIP), de Barcelona.

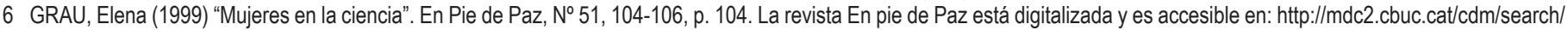
collection/piedepaz

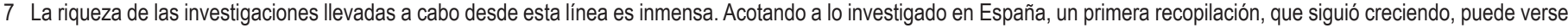
en: ORTIZ; BIRRIEL y MARIN (1998). 
media una profunda revisión crítica. Es decir, resulta difícil admitir que todo conocimiento es situado y que un conocimiento situado en una experiencia de exclusión, es mejor que el anclado en ningún lugar. ${ }^{8}$ Este es pues el apoyo que proporciona la crítica feminista de la ciencia, sustentada por la corriente más amplia de la filosofía de la ciencia que, filosóficamente, minó el positivismo.

En una reseña sobre este libro, realizada para el Journal of Philosophy of Science, ${ }^{9}$ Anna Estany había expresado sus dudas acerca de que existiera una relación entre el marco teórico aportado por las críticas feministas de la ciencia -y que se presenta en el primer capítulo del libro Pioneras españolas en las ciencias- y el desarrollo de la parte histórica del citado libro. El caso es, le escribía a esta filósofa, que sin el esfuerzo dedicado a la lectura de las filósofas feministas de la ciencia, sin la asimilación de los debates y críticas que desde el feminismo se le hacen a la ciencia, y sin la adopción final de un lugar, por mi parte, desde donde llevar a cabo una mirada diferente hacia la historia de la ciencia española del periodo abordado, nunca hubiera podido escribir este libro, ya que mi propia formación positivista recibida en la carrera de Físicas, me hubiera impedido 'ver' y dar importancia a las aportaciones de estas pioneras.

La crítica de las filósofas feministas desveló los sesgos sexistas de la tradición científica (teorías que se han mantenido a lo largo de la historia de la ciencia, como, por ejemplo, que las mujeres eran incapaces de pensar o estudiar por tener mermadas sus capacidades cerebrales, dado que, esgrimían, la energía que utilizaban para la reproducción ¡iba en detrimento del desarrollo cerebral!). Estas teorías, entre otros factores, estaban en la base de la prohibición que les negaba el acceso a las instituciones de la ciencia (Universidades, sociedades científicas...). En su lucha por incorporarse a las instituciones de la ciencia, por ser reconocidas como sujetos del conocimiento, la voz de las mujeres contribuyó a socavar las seguridades de la ciencia -lo incontrovertible de su discurso-.

El punto de vista feminista mantiene que todo el conocimiento es situado y que algunas situaciones sociales son preferibles como punto de partida para

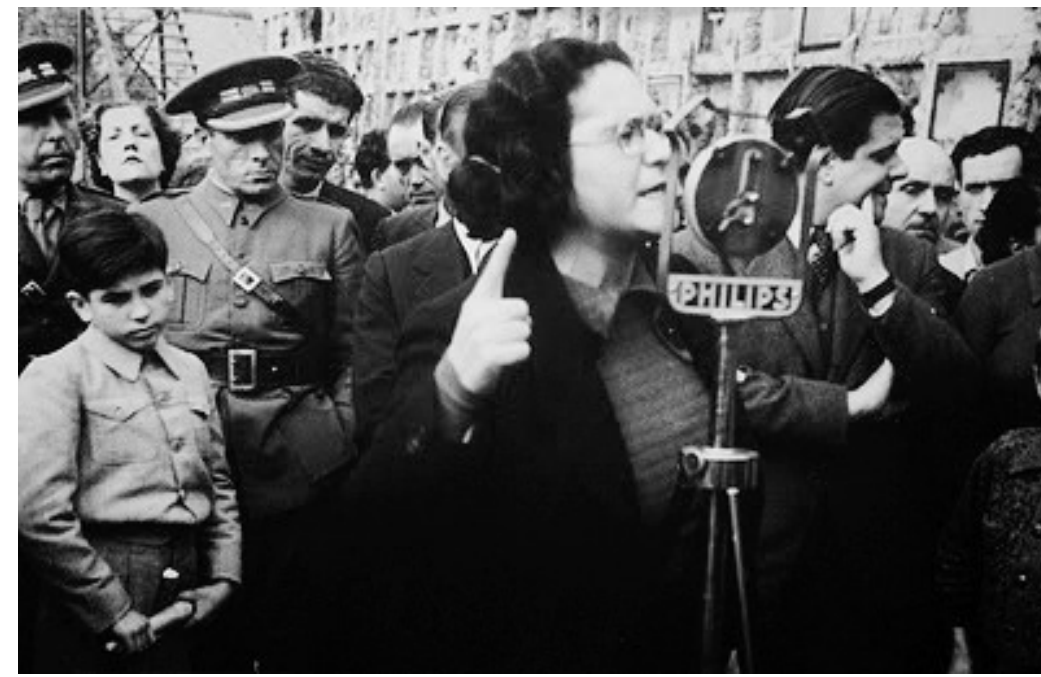

Federica Montseny (1905-1994), fue ministra durante la II República española, siendo la primera mujer en ocupar un cargo ministerial en la Europa Occidental.

construirlo (...) El conocimiento así logrado está saturado de historia y vida social, en lugar de ser abstraído de ellas. Esto no lo distingue del conocimiento elaborado desde otras bases que, aun sin reconocerlo, también está saturado de huellas de las comunidades que lo producen. (...). Por esta vía, un conocimiento situado llega a ser más objetivo que un conocimiento supuestamente neutral. ${ }^{10}$

Hay que ponerse a temblar cuando alguien afirma que habla desde la objetividad. ¿Acaso se puede vivir/actuar/pensar desde fuera del propio lenguaje, la propia cultura, la propia circunstancia histórica? Abogar por la objetividad puede ser un deseo respetable, pero hay que preguntarse si se logra oscureciendo el marco de valores en el que estamos inmersos. Como defiende Sandra Harding, la objetividad no es algo a lo que debamos renunciar, pero no se logra proclamándola sino poniendo sobre la mesa los propios valores: colocando bajo el escrutinio, o en el mismo plano al sujeto y al objeto de la investigación.

Todo conocimiento es situado, no solo espacialmente, también cultural e históricamente, "situación" afecta a los interrogantes que se consideran relevantes para investigar, las hipótesis que se emiten como guías de la investigación, los datos. Estas que se almacenan como relevantes, la organización y

8 Algo que, por otra parte, no es posible. El conocimiento siempre estará asentado en la experiencia vital de la comunidad que lo elabora. Ya sabemos que las comunidades científicas se consideran a sí mismas 'transparentes' para los valores sociales, es decir no los detectan, pero este supuesto es cuestionado desde la historia, la filosofía y la sociología de la ciencia.

9 ESTANY, Anna (2000) Journal of Philosophy of Science, vol. 67, № 3, 551-553.

10 MAGALLÓN PORTOLÉS, Op. Cit., p. 56. 
presentación de los datos, y lo que se considera evidencia para validar una hipótesis. Los valores, los juegos de poder, están incidiendo, amparados en la conocida infradeterminación de las teorías por los datos (de modo muy resumido, esto significa que un conjunto de datos no conduce de manera biunívoca a un único esquema teórico, sino que esos datos pueden ser explicados por distintas teorías). No sólo se ve en las teorías biológicas. Es bien sabido que fue el poder de Newton, presidente de la Royal Society, el que inclinó la balanza para que la teoría corpuscular de la luz se impusiera (al menos por un tiempo) sobre la teoría ondulatoria que proponía Huygens, cuando ambas encajaban en la explicación de la naturaleza de la luz.

Podemos preguntarnos si los criterios de verdad y racionalidad pueden articularse con independencia de los valores e intereses sociales y políticos. Mantengo con Harding que el conocimiento es situado y que por tanto existen perspectivas. También que no existe una realidad preconceptual dada que nos sea accesible, por lo que nuestro conocimiento vendrá marcado por los sistemas conceptuales en los que estamos inmersos. Comparto la idea de que los procesos de construcción de la ciencia son sociales, por tanto teñidos de valores. Que es inevitable que la ciencia refleje los valores de los científicos y de la

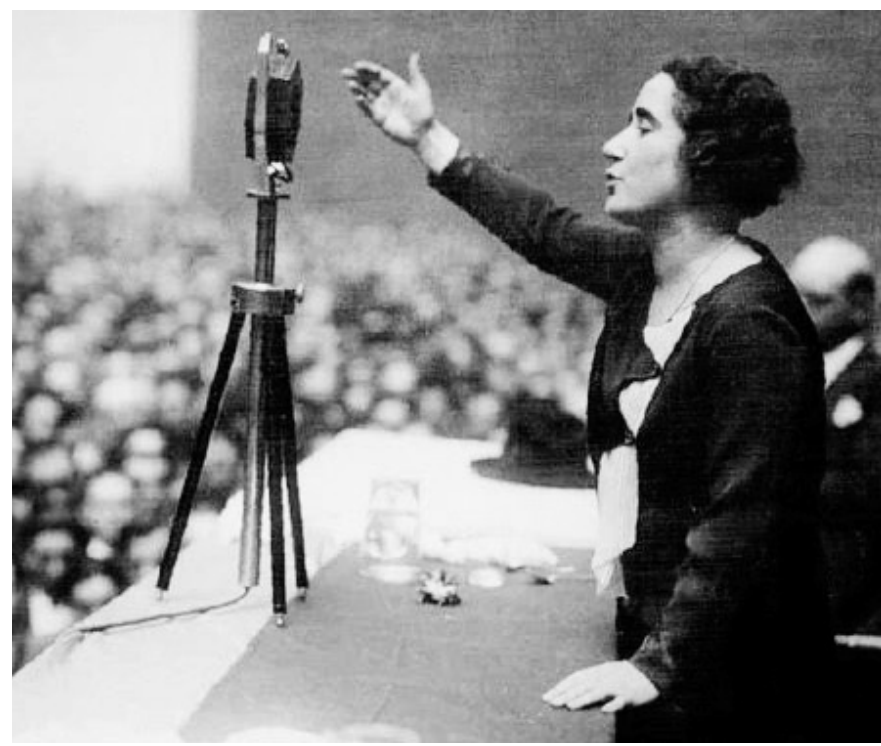

Clara Campoamor (1888 - 1972). Abogada y política madrileña, fue una de las tres diputadas de las cortes constituyentes de la II República y una de las principales precursoras del feminismo en España.

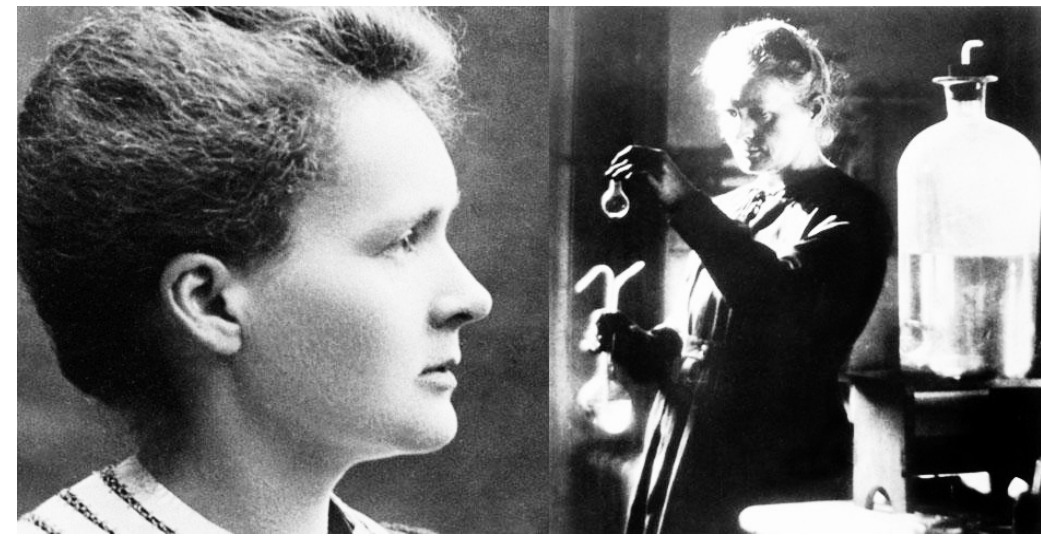

Marie Curie (1867-1934), científica polaca, nacionalizada francesa. Pionera en el campo de la radioactividad. Fue, entre otros méritos, la primera persona en recibir dos Premios Nobel.

sociedad en la que se produce. Ahora bien, como Helen Longino argumenta, creo que sí existen estándares de aceptabilidad racional que son independientes de valores e intereses particulares, aunque no de valores en general.

Lo dicho, no invalida el peso y la importancia de la ciencia y el conocimiento en nuestras vidas. Y, desde luego, "no estamos obligados a escoger entre lo científico y el escepticismo...La tercera posibilidad es aceptar la posición que estamos destinados a ocupar en cualquier caso, la posición de seres que no pueden tener una percepción del mundo que no refleje nuestros intereses y valores, pero que, por todo lo anterior, estamos comprometidos a considerar que algunos puntos de vista sobre el mundo -y, por ello, algunos intereses y valoresson mejores que otros"11. Los valores no se pueden eliminar pero se pueden poner de manifiesto $\mathrm{y}$, añado con Harding, elegir. El conocimiento es más que una actividad individual una actividad social, y este carácter social es a la vez lo que lo convierte en vulnerable de los valores e intereses sociales, pero también lo protege. Como una cuestión social, la ciencia -ya es- pero ha de seguir siendo objeto de polémica.

\section{Contra el determinismo tecnocientífico}

Dando un salto del rescate del pasado al presente y a la proyección sobre el futuro, a las mujeres y otros grupos humanos, que fuimos objeto de discriminación

11 PUTNAM, Hilary (1993) “La objetividad y la distinción ciencia-ética”. En: Martha C. Nussbaum y Amartya Sen (comp.) La calidad de vida. México, Fondo de Cultura Económica, 1996, 193-210, p.209. 
bajo la legitimación de algunas teorías científicas que respondían a los intereses de la masculinidad dominante (sometidas a un determinismo biológico sesgado), nos interesa luchar contra el nuevo determinismo que se perfila: el determinismo tecnocientífico.

La tecnociencia está presente en nuestras vidas, condicionándolas constantemente: el ritmo de nuestros desplazamientos, y por tanto de nuestro vivir los momentos del día, la forma de comunicarnos e informarnos, el ocio, el deporte, el modo de cocinar y el contenido de nuestros alimentos, la relación con nuestro cuerpo y su cuidado están marcados por ella. ¿Alguien nos ha consultado sobre cómo queremos vivir $\mathrm{y}$, en consonancia, qué tipo de tecnociencia queremos desarrollar?

Existen, además, desigualdades marcadas en el acceso a la tecnociencia. Mirando a nuestro alrededor, nos damos cuenta enseguida -a través de la propia TV-, que el complejo tecnocientífico que condiciona al ser humano es desigual a lo largo del planeta. Está ligado a la procedencia cultural y al nivel de ingresos. Algo que se pone de manifiesto en los viajes turísticos o en las intervenciones de ONGs en países en vías de desarrollo. Si un occidental visita un pueblo perdido en el Himalaya, o un cooperante acude a una comunidad remota, va pertrechado de productos y posibilidades (teléfonos móviles, alimentos concentrados, medicamentos, etc., además de un billete de regreso en avión), a menudo inaccesibles para la población visitada. En la coincidencia espacio-temporal de personas marcadas por distintos niveles de acceso tecnológico se ponen de manifiesto, también, las diferencias y desigualdades entre los seres humanos del mundo, desigualdad que acompaña al hecho, más bien fortuito, de haber nacido en un lugar del planeta y con un nivel de renta determinado.

La consciencia de la falta de control sobre la orientación de la tecnociencia y la desigualdad de acceso a ella de las distintas poblaciones, la idea de que el desarrollo constante de la ciencia va en beneficio de la sociedad se encuentra desde hace tiempo deteriorada y en declive. En organismos internacionales como la UNESCO existe preocupación por ese creciente desencuentro entre la ciencia y la sociedad. El prestigio que los ciudadanos y ciudadanas concedían a la ciencia de manera casi generalizada se ha debilitado, minado precisamente por la contundencia y racionalidad de las críticas ecologistas, feministas y las que se llevan a cabo desde los países empobrecidos. Desde estos movimientos se percibe y se critica el papel Hipatia de Alejandría (355-415), legitimador de la ciencia Matemática, filósofa, astrónoma, escritora e ante las prácticas de inventora.

exclusión y dominación a las que se oponen.

Para abordar el desencuentro ciencia-sociedad mencionado, en 1999, la UNESCO convocó una Conferencia Mundial sobre la ciencia, que se celebró en Budapest (26 de Junio al 1 de Julio). La convocatoria fue realizada junto al Consejo Internacional de Uniones Científicas (CIUC), organización que agrupa a miembros de 95 países y de 25 uniones científicas. Bajo el lema, $L a$ Ciencia para el siglo XXI, un nuevo compromiso, se reunieron delegados de 150 países. Allí se debatieron tendencias del desarrollo científico que desde la perspectiva de las necesidades humanas son relevantes y preocupantes: la privatización creciente del saber, la distribución desigual de la tecnociencia en el mundo y la necesidad de introducir el debate ético en el proceso de desarrollo de la ciencia. Hay que decir que, con los años, estas tendencias, sobre todo la mercantilización y privatización del conocimiento y la falta de control democrático sobre la orientación de su desarrollo, se han ido agudizando.

\section{En 1995, Ana María Cetto, escribía en la Revista Española de Física que:}

La ciencia y la tecnología han tenido más éxito en crear nuevos medios para la interacción y comunicación con otros seres humanos $-y$, a veces también, en controlar eficazmente sus vidas- que en entender a esos seres humanos. Se han empleado más exitosamente en desarrollar armas cada vez más complejas que en tratar el problema secular de la guerra y la opresión. El sistema de la ciencia y los científicos enfrentan, por ende,

12 CETTO, Ana María (1995) "La ciudadanía mundial y la formación de los científicos". Revista Española de Física, n 9 (1), 1-2. En esos años, la autora trabajaba en el Instituto de Física de la Universidad Nacional Autónoma de México y era miembro del Comité consultivo del Informe Mundial de la Ciencia, realizado por la UNESCO, en 1998. 
una responsabilidad sin precedentes, pues las aplicaciones de su trabajo pueden tener efectos cada vez más globales sobre un sistema que no está siquiera propiamente entendido. ${ }^{12}$

El desarrollo de la tecnociencia está condicionando la vida humana, bajo la mirada- teoría única que se impone o que alentamos tal vez desde nuestros deseos más nimios por lo que tampoco podemos considerarnos inocentes. El abismo entre los científicos y el resto de la sociedad se agranda. El riesgo está en volver a ser presa de otro determinismo que conforme nuestras vidas por la vía de lo tecnodado. Pero, ¿atañe sólo al científico la responsabilidad de definir nuestro modo de vida? Una pregunta importante que ha de hacernos dirigir la atención hacia la diversidad de fuentes en las que arraiga o puede enraizarse el determinismo, la homogeneidad o la vitalidad de lo diverso. En un mundo donde hemos dejado de creer en las causas lineales y admitimos la complejidad del flujo de corrientes y campos de fuerza que nos tensionan, los desarrollos de la ciencia no se acrisolan en vasijas aisladas de su ambiente; se alimentan de imágenes y sueños, que fueron tal vez convertidos en traza material por un novelista, avanzados por un pensador o escondidos implícitamente en un concepto filosófico capaz de desplegarse y resonar en otros ámbitos. Lo que somos está amasado con los deseos y las proyecciones de pasado y futuro que también crecen en las creaciones literarias y de pensamiento. Nadie hace ciencia sin una metáfora implícita que le sustente. Por todo lo anterior, urge que la orientación de la ciencia pase a la agenda democrática.

\section{Referencias bibliográficas}

- BARRAL, M.J; MAGALLÓN, C.; MIQUEO, C. y SÁNCHEZ, D. (eds.) (1999) Interacciones ciencia y género: discursos y prácticas científicas de mujeres, Barcelona, Icaria-Antrazyt.

- CETTO, Ana María (1995) “La ciudadanía mundial y la formación de los científicos". Revista Española de Física, $\mathrm{N}^{\circ} 9$ (1), 1-2.

- ESTANY, Anna (2000) Reseña del libro Pioneras españolas en las ciencias: las mujeres del Instituto Nacional de Física y Química, de Carmen Magallón Portolés. Journal of Philosophy of Science, vol. 67, $\mathrm{n}^{\circ}$ 3, 551-553.

- GRAU, Elena (1999) "Mujeres en la ciencia”. En Pie de Paz, no 51, 104-106.
- HARDING, Sandra (1986) The Science Question in Feminism. Ithaca, NY, Cornell University Press.

- HARDING, Sandra (ed.)(1987) Feminism and Methodology. Bloomington, Indiana University Press.

- HARDING, Sandra (1991) Whose Science? Whose Knowledge? Thinking from Women's Lives. Ithaca, NY, Cornell University Press.

- HARDING, Sandra (1997) “Women's Standpoints on Nature. What Makes Them Possible?" En: Sally Gregory Kohlstedt y Helen E. Longino (eds.) Women, Gender and Science: New Directions. Ithaca, N.Y., Osiris. A Research Journal devoted to the History of Science and its Cultural Influences, 186-200.

- HEKMAN, Susan (1997) "Truth and Method: Feminist Standpoint Theory Revisited". Signs: Journal of Women in Culture and Society,22(2), 341365.

- MAGALLÓN PORTOLÉS, Carmen (1998) Pioneras españolas en las ciencias. Las mujeres del Instituto Nacional de Física y Química. Madrid, CSIC.

- MAGALLÓN PORTOLÉS, Carmen (2001) “Ciencia, pensamiento y necesidades humanas: una reflexión desde la responsabilidad". En: Seminario de Investigación para la paz (ed.) La paz es una cultura. Zaragoza, Gobierno de Aragón, 127-150.

- ORTIZ GOMEZ, Teresa; BIRRIEL SALCEDO, Johanna y MARIN PARRA, Vicenta (1998) Universidad y feminismo en España (I). Bibliografía de estudios de las mujeres (1992-1996). Granada, Universidad de Granada, colección Feminae, nueva etapa, No3.

- PUTNAM, Hilary (1993) “La objetividad y la distinción ciencia-ética". En: Martha C. Nussbaum y Amartya Sen (comp.) La calidad de vida. México, Fondo de Cultura Económica, 1996.

- ROSSITER, Margaret W. (1982) Women Scientists in America. Struggles and Strategies to 1940. Baltimore/ London, The Johns Hopkins University Press.

- ROSSITER, Margaret W. (1995) Women Scientists in America. Before Affirmative Action, 1940-1972. Baltimore/London, The Johns Hopkins University Press. 